Chirurg 2020 $91: 431$

https://doi.org/10.1007/s00104-020-01149-z Online publiziert: 21. Februar 2020

(c) Springer Medizin Verlag $\mathrm{GmbH}$, ein Teil von Springer Nature 2020

\section{Originalpublikation}

Nicholas LS, Wee I, Shabbir A, Kim G, So JBK (2019) Pouch versus no pouch following total gastrectomy: meta-analysis of randomized and non-randomized studies. Ann Surg 269:1041-1053

Hintergrund und Fragestellung. In der aktuellen Fassung der S3-Leitlinie „Magenkarzinom“ von 2019 ist festgehalten, dass es keinen allgemein anerkannten Standard zur Rekonstruktion nach Gastrektomie gibt. Gegenwärtig ist die $\mathrm{Re}$ konstruktion mittels ausgeschalteter jejunaler Schlinge mit einfacher Roux-YÖsophagojejunostomie die am häufigsten angewandte Technik, die jedoch mit erheblichen funktionellen Problemen assoziiert ist. Die postoperative klinische Symptomatik dieser Rekonstruktion ist Folge eines Verlustes des gastralen Reservoirs, der gastrointestinalen vagalen Stimulation, der gastralen sekretorischen Funktion sowie der gastroösophagealen Sphinkterfunktion. In einer aktuell publizierten Metaanalyse wurde die Frage der alternativen Pouch-Rekonstruktion untersucht.

Methoden. In diese Metaanalyse wurden nach Literatursuche 17 prospektivrandomisierte und 8 Beobachtungsstudien mit insgesamt 1621 Patienten aus dem Zeitraume von 1987 bis 2017 eingeschlossen.

Ergebnisse. Auch wenn die Operationszeit für die Pouch-Rekonstruktion im Mittel 24 min länger dauerte, waren die postoperative Komplikationsrate einschließlich Anastomoseninsuffizienz

W. Schröder · C. J. Bruns

Klinik für Allgemein-, Viszeral- und Tumorchirurgie, Universitätsklinik Köln, Köln, Deutschland

\title{
Rekonstruktion nach Gastrektomie - Pouch oder kein Pouch?
}

sowie Krankenhausliegedauer und -mortalität für beide Gruppen vergleichbar. Auf der anderen Seite führte die PouchRekonstruktion zu einer deutlichen Reduktion der Dumping-Symptomatik nach 3 bis 6 Monaten (8,1\% vs. $32,4 \%$, Risiko Ratio (RR) 0,36, $95 \%$-Konfidenzintervall (CI) $0,21-0,60$ ) und 12 bis 24 Monaten (2,8\% vs. $23,6 \%$, RR 0,27, CI 0,16-0,46). Im Langzeitverlauf nach 12 und 24 Monaten wurden weiterhin eine geringere Rate an Ösophagitis/ Sodbrennen, Dumping-Syndrome und Probleme bei Nahrungsaufnahme beobachtet $(63,73$ und $50 \%$ relative Reduktion). Nach diesen beiden Zeitintervallen war auch der Serumalbuminspiegel (40,5 vs. 37,9g/l) und der Body-Mass-Index (BMI, 22,2 vs. 20,9) bei Patienten mit Pouch signifikant höher.

\section{Kommentar}

Die aktuelle Metaanalyse zeigt eindeutige funktionelle Vorteile für die Rekonstruktion mittels Pouch, ohne dass die Komplikationsrate nach Gastrektomie erhöht wird. Die Autoren dieser Arbeit folgern, dass es eigentlich keinen Grund gibt, Patienten nach Gastrektomie ohne Pouch zu versorgen. Mit diesen Ergebnissen wird die Diskussion um die gastrale Rekonstruktion wieder zu neuem Leben erweckt. Unbeantwortet bleibt jedoch die Frage, welche der vielen möglichen Pouch-Formen und welches Pouch-Volumen die besten Ergebnisse zeigen. Im Zeitalter minimal-invasiver Techniken wird damit aber auch die Lernkurve der Gastrektomie noch einmal verlängert.

\section{Korrespondenzadresse}

Prof. Dr. W. Schröder, FACS, FEBS

Klinik für Allgemein-, Viszeral- und

Tumorchirurgie, Universitätsklinik Köln Kerpener Str. 62, 50937 Köln, Deutschland wolfgang.schroeder@uni-koeln.de

Interessenkonflikt. W.Schröder und C.J. Bruns geben an, dass kein Interessenkonflikt besteht. 